\title{
Isolation and Characterization of a Type II Peroxiredoxin Gene from Panax ginseng C. A. Meyer
}

\author{
Yu-Jin Kim, Jung-Hye Lee, Ok Ran Lee, Ju-Sun Shim, Seok-Kyu Jung, Na-Ri Son, Ju-Han Kim, \\ Se-Young Kim, and Deok-Chun Yang*
}

Korean Ginseng Center for Most Valuable Products and Ginseng Genetic Resource Bank, Kyung Hee University, Suwon 449701, Korea

A peroxiredoxin cDNA (PgPrx) was isolated and characterized from the leaves of Panax ginseng. The cDNA is 716 nucleotides long and has an open reading frame of 489 base pairs with a deduced amino acid sequence of 162 residues. The calculated molecular mass of the mature protein is approximately $17.4 \mathrm{kDa}$ with a predicted isoelectric point of 5.37 . A GenBank BlastX search revealed that the deduced amino acid sequence of PgPrx shares a high degree homology with type II peroxiredoxin (Prx) proteins in other plants. The PgPrx gene was highly expressed in leaves, and expressed at a low level in the stem. To analyze the gene expression of PgPrx in response to various abiotic stresses, we utilized real-time quantitative RTPCR. Our results reveal that $\operatorname{Pg} \operatorname{Prx}$ expression is induced by ultraviolet irradiation, low temperature, and salt. The induction of PgPrx in response to abiotic stimuli suggests that ginseng Prx may function to protect the host against environmental stresses.

Keywords: Ginseng, Environmental stresses, Peroxiredoxin gene, RT-PCR

\section{INTRODUCTION}

Exposing plants to unfavorable environmental conditions such as low or elevated temperature, ultraviolet (UV) irradiation, or salt stress can induce the production of reactive oxygen species (ROS) such as the superoxide radical, hydrogen peroxide, and the hydroxyl radical. ROS cause serious damage to biological macromolecules. To protect against these toxic oxygen intermediates, plants use various enzymatic and non-enzymatic defense mechanisms that prevent or repair oxidative damage [1].

Peroxiredoxins (Prxs) constitute the most recently identified group of $\mathrm{H}_{2} \mathrm{O}_{2}$-decomposing antioxidant enzymes. Prxs are present in all organisms, from prokaryotes to eukaryotes, and they catalyze the reduction of various hydroperoxides into the corresponding alcohol or water [2-4]. Prxs are thiol-based peroxidases that pos- sess at least one conserved cysteine in their N-terminus that reacts with peroxide substrates [5]. In addition to the reduction of $\mathrm{H}_{2} \mathrm{O}_{2}$, Prx proteins also detoxify alkyl hydroperoxides and peroxinitrite, despite the fact that significant differences exist in substrate specificity and kinetic properties [2]. Experimental evidence for Prx function in plants has revealed multiple mechanisms: it can serve as an antioxidant, a modulator of cell signaling pathways, and as a redox sensor [2]. Four Prx subgroups have been described in plants, and they are distinguished by the number and position of the conserved cysteine residues: 1-Cys Prx, 2-Cys Prx, type II Prx, and Prx Q [2,6]. 1-Cys Prx is a dormancy-related protein [7] that is localized in the nucleus, where it helps to protect macromolecules from oxidative damage. 2-Cys Prx is a

\footnotetext{
(c) This is an Open Access article distributed under the terms of the Creative Commons Attribution Non-Commercial License (http://creativecommons.org/licenses/by-nc/3.0/) which permits unrestricted non-commercial use, distribution, and reproduction in any medium, provided the original work is properly cited.
}

Received 5 Jul. 2010, Revised 23 Aug. 2010, Accepted 24 Aug. 2010

*Corresponding author

E-mail: dcyang@khu.ac.kr

Tel: +82-31-201-2100, Fax: +82-31-205-2688 
nuclear-encoded protein targeted to the chloroplast, where it protects the photosynthetic membrane from oxidative damage [8]. Type II Prx was isolated as a target of cytoplasmic thioredoxin-h, and displays antioxidant activity in Arabidopsis thaliana [9]. Finally, Prx Q was identified initially in Sedum lineare as a homolog of the Escherichia coli bacterioferritin co-migratory protein [3]. Among the four groups, the type II Prxs are the least studied with respect to peroxidase activity, although it has recently been observed in yeast [9,10], Arabidopsis [6,9,11], poplar [4], and Chinese cabbage [12]. At least in Arabidopsis, the type II Prx is complex and composed of several different members. Six Prx II genes have been identified in Arabidopsis [6,11], one of them seems to be a pseudogene; the others are expressed in leaves. Three Prxs localized to the cytosol (AtPrx II B, C, and D), one to the chloroplast (AtPrx II E), and one to the mitochondria (AtPrx II F) $[6,13,14]$.

Although the molecular and biochemical characterizations of $\operatorname{Prx}$ genes in plants are advancing rapidly, the functional role of the type II Prx remains poorly defined. To the best of our knowledge, no research to date has examined Prx in ginseng. Korean ginseng (Panax ginseng $[P$. ginseng $]$ C. A. Meyer) is a perennial herb in the family Araliaceae. It has been cultivated for its highly valued root, which is used for medicinal purposes [15]. Despite its medicinal importance, little genomic analysis has been carried out on this plant. In the present study, the isolation and characterization of a peroxiredoxin cDNA isolated from $P$. ginseng, as well as its possible role in resisting various abiotic stresses, is reported.

\section{MATERIALS AND METHODS}

\section{Plant materials and growth conditions}

The specific type of ginseng used was $P$. ginseng cv. "Hwang-Sook (yellow berry variant)" (Ginseng Genetic Resource Bank, Suwon, Korea). Cultured 3-week-old plantlets were used for treatments and nucleic acid extractions, as described previously [16]. For analysis of gene expression in different organs, samples were collected from leaves, roots, and stems of 6-week-old $P$. ginseng plantlets. Plants were grown in a growth room at $25^{\circ} \mathrm{C}$ with a $16 \mathrm{~h}$ photoperiod.

\section{RNA purification and construction of a CDNA library}

Total RNA was isolated from leaves of 4-year-old ginseng plants, using the aqueous phenol extraction procedure described by Morris et al. [17]. Poly (A) ${ }^{+}$RNA was isolated using an oligo (dT) cellulose column and the Poly (A) Quick mRNA isolation kit (Stratagene, La
Jolla, CA, USA). A commercial cDNA synthesis kit was used to construct a cDNA library according to the manufacturer's instructions (Clontech, Palo Alto, CA, USA). Size-selected cDNA was ligated into the $\lambda$ TriplEx 2 vector and packaged in vitro using a Gigapack III Gold Packaging Extract kit (Stratagene).

\section{Nucleotide sequencing and sequence analysis}

Homologous sequences of Prx expressed sequence tags (EST) were compared against the GenBank databases using a BLASTX algorithm. Nucleotide and amino acid sequence analyses were performed using the DNASIS program (Hitachi Software Engneering America Ltd., CA, USA). Deduced amino acid sequences were used to search for homologous proteins in the databases using the NCBI BLAST search engine. We used ClustalX with default gap penalties to perform multiple alignment of Prx isolated from ginseng with previously registered homologs in other species. Based on this alignment, a phylogenetic tree was constructed according to the neighbor-joining method using the MEGA3 program. Bootstrap analysis with 1,000 replicates was also conducted to obtain confidence levels for the branches. Secondary structure was determined by SOPMA program, and the hydropathy pattern was analyzed in a manner described by Kyte and Doolittle [18]. Protein properties were estimated using ProtParam [19].

\section{Abiotic stress treatment}

For chemical stress or plant hormone treatments, the plantlets were placed in Murashige and Skoog medium containing $100 \mathrm{mM} \mathrm{NaCl}$ for various periods. Chilling stress was applied by exposing the plantlets to a $4^{\circ} \mathrm{C}$ environment. For the UV treatment, the plantlets were irradiated under UV lamps at $1.35 \mu \mathrm{E} \mathrm{m}^{-2} \mathrm{~s}^{-1}$ (below $280 \mathrm{~nm}$ ). Control and stress-exposed plant materials were immediately frozen in liquid nitrogen and stored at $-70^{\circ} \mathrm{C}$ until used.

\section{Real-time quantitative RT-PCR (qRT-PCR)}

Total RNA was extracted from plantlets of $P$. ginseng using the RNeasy mini kit (Qiagen, Valencia, CA, USA). To perform RT-PCR, $5 \mu \mathrm{g}$ total RNA was used as a template for reverse transcription using oligo $(\mathrm{dT})_{15}$ primer $(0.2 \mathrm{mM})$ and AMV Reverse Transcriptase (10

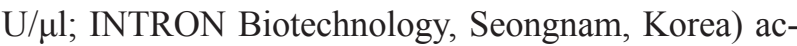
cording to the manufacturer's instructions. qRT-PCR was performed using $10 \mathrm{ng}$ cDNA in a $10 \mu \mathrm{L}$ reaction volume with $\mathrm{SYBR}^{\circledR}$ Green SensimixPlus Master Mix (Quantace, Watford, England). Specific primers for $\operatorname{PgPrx}, 5^{\prime}$-TTCCTAGCTGACGGATCAGG-3' and 
5'-GAATTCCCCACCAGTCTCAA-3', were used. The thermal cycler conditions recommended by the manufacturer were used as follows: $10 \mathrm{~min}$ at $95^{\circ} \mathrm{C}$, followed by 40 cycles of $95^{\circ} \mathrm{C}$ for $10 \mathrm{~s}, 58^{\circ} \mathrm{C}$ for $10 \mathrm{~s}$, and $72^{\circ} \mathrm{C}$ for $20 \mathrm{~s}$. The fluorescent product was detected in the last step of each cycle. Amplification, detection, and data analysis were performed using a Rotor-Gene 6000 real-time rotary analyzer (Corbett Life Science, Sydney, Australia). The threshold cycle $\left(\mathrm{C}_{t}\right)$ represents the number of cycles during which the fluorescence intensity is significantly higher than the background fluorescence during the initial exponential phase of PCR amplification. To determine the relative fold differences in template abundance for each sample, the $C_{t}$ value for $\operatorname{PgPrx}$ was first normalized to the $\mathrm{C}_{\mathrm{t}}$ value for $\beta$-actin, and relative differences were calculated using the formula $2^{-\Delta \Delta C t}$. Three independent experiments were performed.

\section{RESULTS}

\section{Isolation and amino acid sequence analysis of $P g P r x$}

From our EST analysis of a cDNA library that was prepared from the leaves of 4-year-old field-grown $P$. ginseng, we identified a cDNA clone encoding a peroxiredoxin gene. This gene is named PgPrx (P. ginseng Prx), and its sequence has been deposited in GenBank. The obtained PgPrx cDNA is 716 nucleotides long and contains a putative open reading frame (ORF) of 489 base pairs. This ORF encodes a peroxiredoxin protein with 162 amino acids, beginning at the initiation codon ATG (position 98) and ending at the stop codon TAA (position 584). The aliphatic index, which is regarded as a positive factor that increases thermostability, was calculated to be 94.51, using ProtParam [19]. In the deduced amino acid sequence of the $P g P r x$ protein, the total number of negatively charged residues (aspargine+glutamin) was 22, while the total number of positively charged residues (arginine+lysine) was 18 . The instability index was computed to be 32.85 , which classifies the protein as stable. The molecular mass of the mature protein was calculated to be approximately $17.4 \mathrm{kDa}$, with a predicated isoelectric point (pI) of 5.37.

\section{Homology analysis}

A GenBank BlastX search revealed that the deduced amino acid of PgPrx shares a high degree of homology with Prx of the Citrus hybrid cv. Shiranuhi (ABL67649; $83 \%$ identity, 93\% similarity), N. nucifera (ABN46981; $83 \%$ identity, 91\% similarity), Plantago major (AH58634; $82 \%$ identity, 90\% similarity), and Capsicum annuum (AAL35363.2; 77\% identity, 90\% similarity). The com- parison also showed strong homology with the type II Prx of Lycopersicon esculentu (L. esculentu; AAP34571; $76 \%$ identity, $90 \%$ similarity) and Xerophyta viscose (ACA13182; 75\% identity, 90\% similarity) (Fig. 1A). A phylogenetic study comparing the $\operatorname{Pg} P r x$ amino acid sequence to the sequences of the previously reported four types of proteins [17] led to the conclusion that $P g P r x$ is a Prx II (Fig. 1B). In addition, in BlastP searches, PgPrx had high score hits when compared to the Prx 5 family and PrxII; indeed, PrxII is homologous with mammalian $\operatorname{Prx} 5$ [17].

The hydropathy pattern of $\operatorname{PgPrx}$ is similar to that of other homologous Prxs (Fig. 2A). Secondary structure analysis and molecular modeling of PgPrx were performed using SOMPA. Secondary structure analysis revealed that $\operatorname{PgPrx}$ consists of $48 \alpha$-helices, $15 \beta$-turns joined by 39 extended strands, and 60 random coils. The Citrus hybrid displays almost the same secondary structure as $\operatorname{Pg} \operatorname{Prx}$ : $48 \alpha$-helices, $11 \beta$-turns joined by 42 extended strands, and 61 random coils. The other homologous plant Prxs including PrxII of L. esculentu also showed a similar structure (Fig. 2B).

\section{Organ-specific expression patterns of $\mathbf{P g P r x}$}

The expression patterns of $P g P r x$ in different $P$. ginseng organs were examined using real time qRT-PCR. $P g P r x$ was highly expressed in the leaf and root, with relative low levels of expression in the stem (Fig. 3).

\section{Temporal expression of PgPrx in response to abiotic stresses}

To elucidate the expression patters of $\operatorname{PgPrx}$ in response to stressors such as UV, chilling, and salt, realtime qRT-PCR analysis was performed. Fig. 4A shows the expression pattern of $\operatorname{PgPrx}$ in response to salt stress (Fig. 4A). Salinity stress $(100 \mathrm{mM} \mathrm{NaCl})$ caused a marked (8-fold) and immediate (1 h) induction of PgPrx; however, transcription levels then decreased to 2 -fold or remained at a similar level as controls for the subsequent $72 \mathrm{~h}$. UV exposure also caused a dramatic and rapid induction of $\operatorname{PgPrx}$, inducing transcript levels to increase by 64 -fold $1 \mathrm{~h}$ post-treatment, and reaching a maximal induction (87-fold) in $4 \mathrm{~h}$; thereafter, it decreased dramatically (Fig. 4B). Under chilling stress, $\operatorname{Pg} \operatorname{Prx}$ mRNA level seemed to have been slightly suppressed initially (0.67-fold $1 \mathrm{~h}$ post-treatment), but then increased by 10 fold within $4 \mathrm{~h}$, and continued to be higher $8 \mathrm{~h}$ and $24 \mathrm{~h}$ later (15-fold and 64-fold, respectively). However, 48 hours after treatment, $\operatorname{Pg} \operatorname{Prx}$ expression was 10-fold lower; 72 hours post-treatment, it was 7-fold lower (Fig. 4C). 

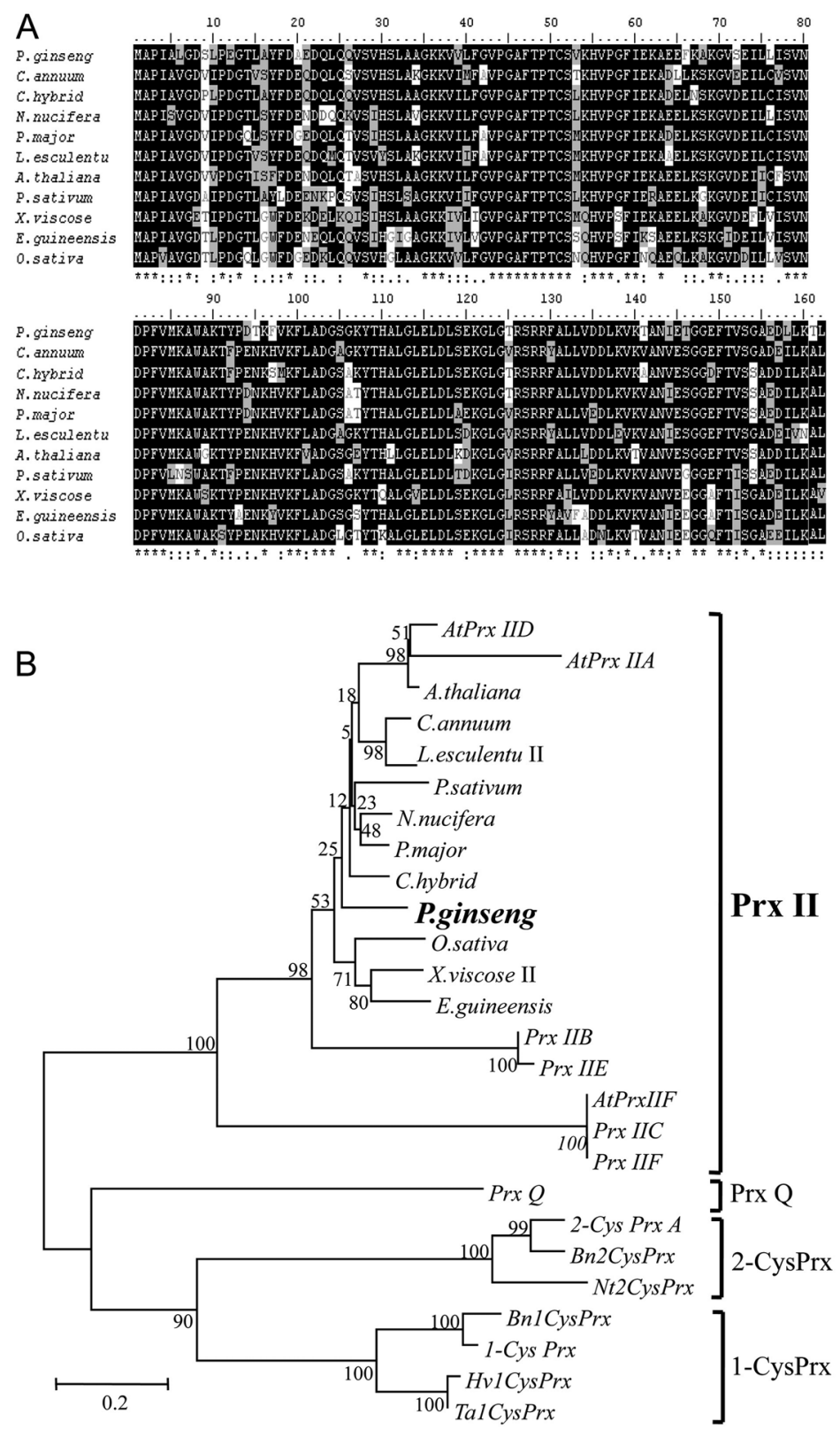

Fig. 1. Sequence homology and phylogenetic analysis of $P g P r x$ compared to other peroxiredoxin proteins. (A) Comparison of the putative amino acid sequences of PgPrx and those of Prx II genes from the following plants: Capsicum annuum (C. annuum, AAL35363.2), Citrus hybrid (C. hybrid) cv. Shiranuhi (ABL67649), Nelumbo nucifera (N. nucifera, ABN46981), Plantago major (P. major, AH58634), Lycopersicon esculentu (L. esculentu, AAP34571), Arabidopsis thaliana (A. thaliana, NP_176773), Pisum sativum (P. sativum, CAQ56034), Xerophyta viscose type II (X. viscose, ACA13182), Elaeis guineensis (E. guineensis, ACF06569), and Oryza sativa (O. sativa, NP_001043845). Hyphens within amino acid sequences denote a gap. Shaded boxes indicate well-conserved residues, * represents a conserved amino acid and : represents a very similar amino acid. (B) Phylogenic comparison of peroxiredoxin (Prx) from Panax ginseng (P. ginseng) and the four types of peroxiredoxin protein from other plants. The analysis is based on the deduced amino acid sequences of Prx genes from various species. The neighbor-joining method was used and the branch lengths are proportional to divergence, where a scale of 0.1 represents $10 \%$ change.

\section{DISCUSSION}

Here we provide the first report of the isolation and characterization of the peroxiredoxin gene in ginseng. BlastX database searches identified Prx sequences in other plant species as the best matches. Analyses of amino acid homology, hydropathy pattern, and secondary structure revealed that $\operatorname{Pg} P r x$ is very similar to other homologous plant Prx genes (Fig. 1). The Prx genes can be divided into four subgroups: 1-Cys Prx, 2-Cys Prx, type II Prx, and Prx Q. Database searches suggested the PgPrx belongs to the type II family. All Prx molecules 
A

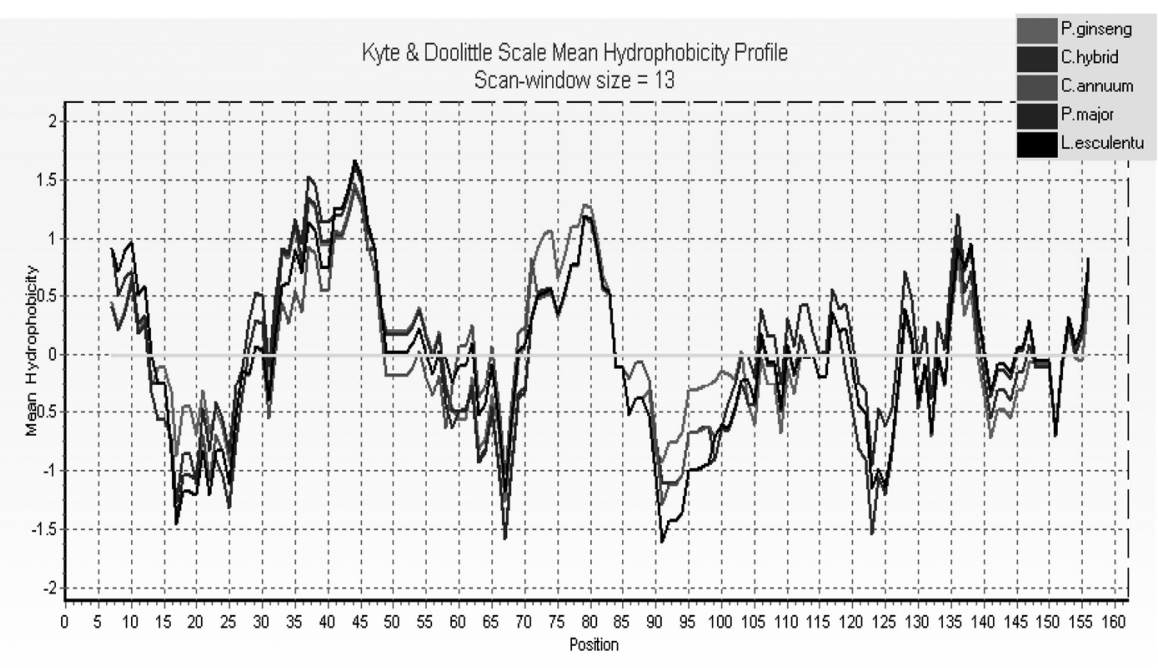

$\mathrm{B}$

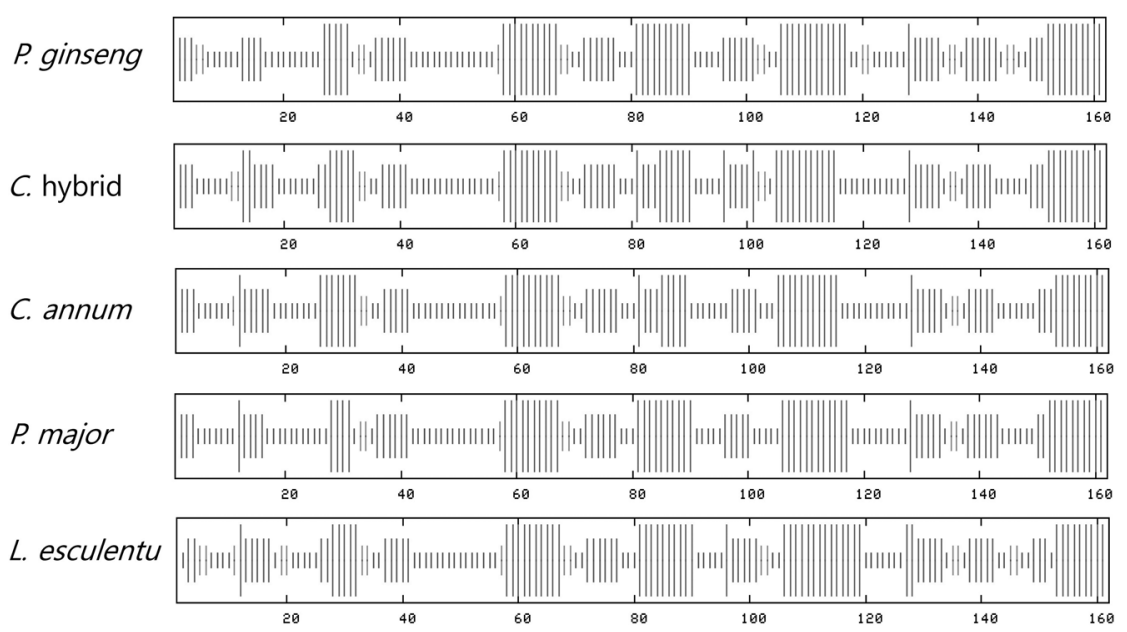

Fig. 2. Superimposed hydrophilicity profiles and secondary structure predictions for peroxiredoxin. (A) Hydropathic index analysis. Hydrophobic domains are indicated by positive numbers; hydrophilic domains are above the line and hydrophilic domains are below it. Citrus hybrid (C. hybrid) cv. Shiranuhi, Capsicum annuum (C. annuum), Plantago major (P. major), and Lycopersicon esculentu (L. esculentu). The helix, sheet, turn, and coil structures are indicated in order from the longest to the shortest. (B) Comparison of the secondary structures of PgPrx, C. hybrid, C. annuum, $P$. major, and $L$. esculentu by SOPMA.

have a similar basic protein structure containing a thioredoxin fold, and their molecular masses range between 17 and $22 \mathrm{kDa}$ [2]. The PgPrx cDNA encodes a Prx protein of 162 amino acids, with a predicted molecular weight of $17.4 \mathrm{kDa}$ and a $\mathrm{pI}$ value of 5.37. Similarly, cytosolic AtPrxII B and C also have masses of $17.4 \mathrm{kDa}$ with pI values of 5.17 and 5.33, respectively [11]. All of the Prx isoforms have conserved-catalytic cysteine (Cys) residues in common. The absolutely conserved Cys is the catalytic or peroxidatic Cys [20,21]. Catalytic Cys residues undergo oxidation during the peroxidereduction reaction, and need to be reduced by electron donors. The active site [6], which neighbors Cys51, is well conserved among PgPrx and homologous plant
Prxs, whereas the second conserved Cys (Cys 76) is only present in some plants (Fig. 1A). The second Cys is also absent from cytosolic Prx from other plants such as $N$. nucifera and $O$. sativa, supporting the fact that this Cys is not absolutely necessary for the protein's functional activity [22]. The transcripts of PgPrx were welldetected in the leaf and root of ginseng plantlets. In the case of Arabidopsis, the expression of Prx II, Prx IIB, and $E$ is most abundantly expressed in green tissue, and Prx IIC and $D$ are highly expressed in the flower and root, whereas Prx IIF is ubiquitously expressed [21]. This suggests that Prx genes are expressed in most organs, but with different organ specificities.

Prx proteins from organelles may prevent DNA dam- 


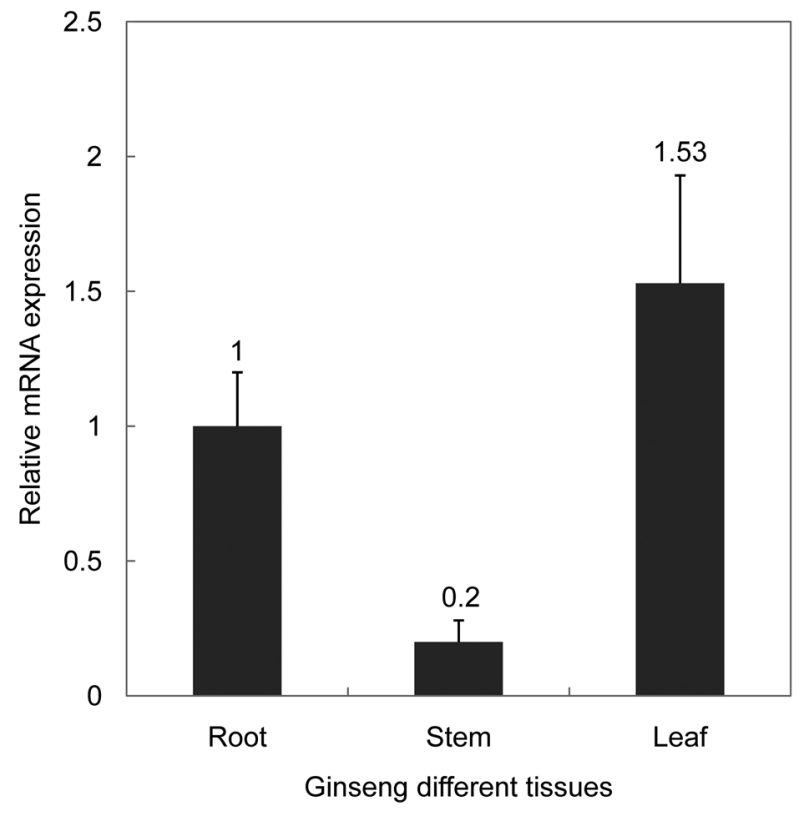

Fig. 3. Relative quantities of PgPrx mRNA in the root, stem, and leaf organs of ginseng plantlets. The error bars represent the standard error of the mean for three independent replicates.

age [2]. A few representatives of the type II Prx have been cloned from plant sources, but knowledge on their molecular functions is limited. Mitochondrial Prx II responds to oxidative stress in Arabidopsis [14], plays a role in plant fungal pathogen interactions in poplar [23], and accumulates in response to cold and heavy metal stress in pea plants [24]. Among six identified AtPrx II genes in Arabidopsis, the expression of cytosolic AtPrx $I I B$ and $I I C$ is induced by oxidative stress and salt stress. Chloroplastic Prx IIE is unregulated after bacterial exposure, and its expression decreases in response to salt stress and increases in response to bright light $[6,11]$. Mitochondrial AtPrx IIF expression is constitutive and is essential for root growth under stress conditions [14]. Mitochondrial AtPrx IIF expression increases in response to biotic rather than abiotic stress [23]. In peas, Prx IIF accumulates in cold- and heavy metal-treated plants [24].

When ginseng plantlets were exposed to stresses, $P g$ Prx expression changed as a function of the exposure time. PgPrx expression seems to be up-regulated by salt, chilling, and UV treatment at early time points. Arabidopsis cytosolic Prx II is also up-regulated by salt, unlike chloroplastic and mitochondrial Prx II [6,11,23]. Prx IIB mRNA levels increase slightly after $6-8 \mathrm{~h}$ and Prx IIC mRNA levels increase dramatically within $2 \mathrm{~h}$ in response to $50 \mathrm{mM} \mathrm{NaCl}$ treatment in Arabidopsis [6]. Salinity causes oxidative stress, and salt tolerance is
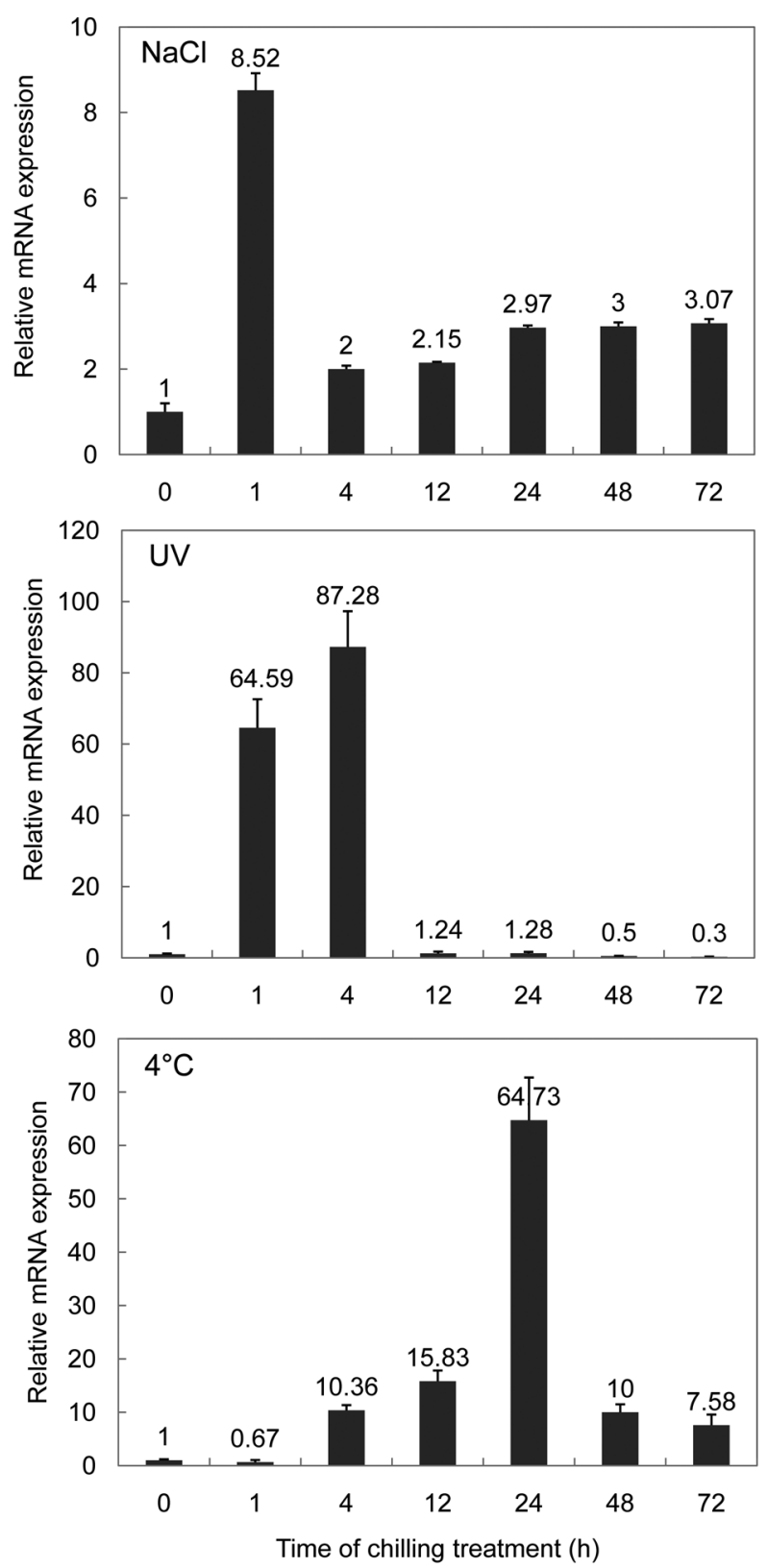

Fig. 4. Relative quantities of PgPrx mRNA in plantlets at various time points post-treatment with various stresses. (A) $100 \mathrm{mM} \mathrm{NaCl}$, (B) ultraviolet light, $(\mathrm{C})$ chilling. The error bars represent the standard error of the mean for three independent replicates.

related to the induction of antioxidant defenses [25]. UV irradiation can cause oxidative stress by generating ROS in plants [26], which leads to oxidative damage. Chilling stress is also involved in the ROS signaling pathway [27]. These conditions trigger oxidative stress and result in the rapid up-regulation of $P g P r x$ gene expression within 1-4 h of exposure. These results may indicate a particular and important role of PgPrx in stress defense. Few studies have considered cytosolic Prx II in plants, 
so further investigations are needed to better understand its mechanisms of action. The present study suggests that Prx II plays an important role in ginseng's defense mechanism against cold, UV, and salinity stresses.

\section{ACKNOWLEDGEMENTS}

This study was supported by a grant from the KGCMVP for Technology Development Program of Agriculture and Forestry, Ministry of Food, Agriculture, Forestry and Fisheries, Republic of Korea.

\section{REFERENCES}

1. Bohnert HJ, Sheveleva E. Plant stress adaptations-making metabolism move. Curr Opin Plant Biol 1998;1:267-274.

2. Dietz KJ, Jacob S, Oelze ML, Laxa M, Tognetti V, de Miranda SM, Baier M, Finkemeier I. The function of peroxiredoxins in plant organelle redox metabolism. J Exp Bot 2006;57:1697-1709.

3. Kong W, Shiota S, Shi Y, Nakayama H, Nakayama K. A novel peroxiredoxin of the plant Sedum lineare is a homologue of Escherichia coli bacterioferritin co-migratory protein (Bcp). Biochem J 2000;351(Pt 1):107-114.

4. Rouhier N, Gelhaye E, Sautiere PE, Brun A, Laurent P, Tagu D, Gerard J, de Fäy E, Meyer Y, Jacquot JP. Isolation and characterization of a new peroxiredoxin from poplar sieve tubes that uses either glutaredoxin or thioredoxin as a proton donor. Plant Physiol 2001;127:1299-1309.

5. Kang SW, Baines IC, Rhee SG. Characterization of a mammalian peroxiredoxin that contains one conserved cysteine. J Biol Chem 1998;273:6303-6311.

6. Horling F, König J, Dietz KJ. Type II peroxiredoxin C, a member of the peroxiredoxin family of Arabidopsis thaliana: its expression and activity in comparison with other peroxiredoxins. Plant Physiol Biochem 2002;40:491-499.

7. Stacy RA, Nordeng TW, Culiáñez-Macià FA, Aalen RB. The dormancy-related peroxiredoxin anti-oxidant, PER1, is localized to the nucleus of barley embryo and aleurone cells. Plant J 1999;19:1-8.

8. Baier M, Dietz KJ. Protective function of chloroplast 2-Cysteine peroxiredoxin in photosynthesis. Evidence from transgenic Arabidopsis. Plant Physiol 1999;119:1407-1414.

9. Verdoucq L, Vignols F, Jacquot JP, Chartier Y, Meyer Y. In vivo characterization of a thioredoxin $\mathrm{h}$ target protein defines a new peroxiredoxin family. J Biol Chem 1999; 274:19714-19722.

10. Jeong JS, Kwon SJ, Kang SW, Rhee SG, Kim K. Purification and characterization of a second type thioredoxin peroxidase (type II TPx) from Saccharomyces cerevisiae.
Biochemistry 1999;38:776-783.

11. Horling F, Lamkemeyer P, König J, Finkemeier I, Kandlbinder A, Baier M, Dietz KJ. Divergent light-, ascorbate-, and oxidative stress-dependent regulation of expression of the peroxiredoxin gene family in Arabidopsis. Plant Physiol 2003;131:317-325.

12. Choi YO, Cheong NE, Lee KO, Jung BG, Hong CH, Jeong JH, Chi YH, Kim K, Cho MJ, Lee SY. Cloning and expression of a new isotype of the peroxiredoxin gene of Chinese cabbage and its comparison to 2Cys-peroxiredoxin isolated from the same lant. Biochem Biophys Res Commun 1999;258:768-771.

13. Kruft V, Eubel H, Jänsch L, Werhahn W, Braun HP. Proteomic approach to identify novel mitochondrial proteins in Arabidopsis. Plant Physiol 2001;127:1694-1710.

14. Finkemeier I, Goodman M, Lamkemeyer P, Kandlbinder A, Sweetlove LJ, Dietz KJ. The mitochondrial type II peroxiredoxin $\mathrm{F}$ is essential for redox homeostasis and root growth of Arabidopsis thaliana under stress. J Biol Chem 2005;280:12168-12180.

15. Nam KY. Clinical applications and efficacy of Korean ginseng (Panax ginseng C. A. Meyer). J Ginseng Res 2002; 26:111-131.

16. Kim YJ, Ham AR, Shim JS, Lee JH, Jung DY, In JG, Lee BS, Yang DC. Isolation and characterization of terpene synthase gene from Panax ginseng. J Ginseng Res 2008; 32:114-119.

17. Morris PC, Kumar A, Bowles DJ, Cuming AC. Osmotic stress and abscisic acid induce expression of the wheat Em genes. Eur J Biochem 1990;190:625-630.

18. Kyte J, Doolittle RF. A simple method for displaying the hydropathic character of a protein. J Mol Biol 1982;157: 105-132.

19. Gasteiger E, Hoogland C, Gattiker A, Duvaud S, Wilkins MR, Appel RD, Bairoch A. Protein identification and analysis tools on the ExPASy server. In: Walker JM, ed. The proteomics protocols handbook. Totowa: Humana Press, 2005. p.571-607.

20. Rouhier N, Jacquot JP. The plant multigenic family of thiol peroxidases. Free Radic Biol Med 2005;38:1413-1421.

21. Gama F, Bréhélin C, Gelhaye E, Meyer Y, Jacquot JP, Rey P, Rouhier N. Functional analysis and expression characteristics of chloroplastic Prx IIE. Physiol Plant 2008;133:599610.

22. Cha MK, Hong SK, Lee DS, Kim IH. Vibrio cholerae thiol peroxidase-glutaredoxin fusion is a 2-Cys TSA/AhpC subfamily acting as a lipid hydroperoxide reductase. $\mathrm{J}$ Biol Chem 2004;279:11035-11041.

23. Gama F, Keech O, Eymery F, Finkemeier I, Gelhaye E, Gardestrom P, Dietz KJ, Rey P, Jacquot JP, Rouhier N. 
The mitochondrial type II peroxiredoxin from poplar. Plant Physiol 2007;129: 196-206.

24. Barranco-Medina S, Krell T, Finkemeier I, Sevilla F, Lázaro JJ, Dietz KJ. Biochemical and molecular characterization of the mitochondrial peroxiredoxin PsPrxII F from Pisum sativum. Plant Physiol Biochem 2007;45:729-739.

25. Shalata A, Mittova V, Volokita M, Guy M, Tal M. Response of the cultivated tomato and its wild salt-tolerant relative Lycopersicon pennellii to salt-dependent oxidative stress: The root antioxidative system. Physiol Plant 2001;112:487-494.

26. Green R, Fluhr R. UV-B-Induced PR-1 Accumulation is mediated by active oxygen species. Plant Cell 1995;7:203212.

27. Einset J, Winge P, Bones A. ROS signaling pathways in chilling stress. Plant Signal Behav 2007;2:365-367. 